\title{
An Unequal Clustering Algorithm with Load Balance for Wireless Sensor Networks
}

\author{
Yinliang $\mathrm{Jia}^{1 *}$, Kangwu Liang ${ }^{1}$ and Chiyu Zhang ${ }^{1}$ \\ ${ }^{1}$ College of Automation Engineering, Nanjing University of Aeronautics and Astronautics, Nanjing 210016 \\ ${ }^{*}$ Corresponding author
}

\begin{abstract}
Good clustering algorithm can effectively reduce the energy consumption of a wireless sensor network (WSN). The random in clustering will reduce the performance of clustering algorithm. Aiming at this problem, this paper proposes an unequal clustering algorithm with load balance (UCLB) for WSN UCLB is a multi-hop algorithm and divides the nodes into clusters of different sizes. Considering the two objectives of load balancing and reducing energy consumption, UCLB elects cluster heads according to a cost parameter that is calculated by the number of around nodes and the distances to these nodes. UCLB preferentially select the node with moderate distances to the elected cluster heads as cluster head to distribute cluster heads evenly in networks. Simulation results show that the algorithm can effectively reduce the network energy consumption and prolong the network lifetime.
\end{abstract}

Keywords-wireless sensor networks; energy efficiency; clustering; load balancing; network lifetime

\section{INTRODUCTION}

WSNs are systems composed of a large number of sensor nodes distributed in the space. The sensor nodes collect information in the monitoring region [1]. WSNs do not need to configure the network infrastructure and provide fast network deployment scheme for national defense, national security, environmental monitoring, traffic management, antiterrorism, emergency communication and so on [2]. WSNs have broad application prospects. Since it is difficult to add energy to the sensor nodes, it is necessary to reduce the energy consumption of sensor nodes in some way, thus prolonging the lifetime of WSNs.

Clustering is one of the effective management of the energy consumption to improve the performance of WSNs. A clustering algorithm includes the election of cluster heads and the communication between a cluster head and the base station, between two cluster heads, between a cluster head and a node [3]. According to the number of hops from a cluster head to the base station, the cluster structure can be divided into single hop network and multi-hop network. In the single hop communication, the base station and cluster heads communicate directly, long-distance communication will make the cluster head energy consume rapidly, so the cluster heads far from the base station die quickly. In a multi-hop communication mode, the information is transmitted from one cluster head to another and transmitted to the base station at last. Of course, such practice has increased the energy consumption of cluster heads near the base station, but compared with the single hop mode, the multi-hop mode can reduce the energy consumption in the network relatively [5].
We propose a novel multi-hop clustering algorithm for WSNs. The rest of the paper is as follows. In Sect. 2, some related algorithms are analyzed. Sect. 3 our algorithm is introduced in detail. Our algorithm and three related algorithms are analyzed and compared by simulation in Sect. 4. We give some conclusion and prospect in Sect. 5.

\section{RELATED WORKS}

The clustering algorithms divide the network into several small clusters which contain many nodes. Each cluster has a cluster head which receives the data sent by the nodes in the cluster and fuses the data before sending to the base station. The clustering algorithms can decrease the amount of data in the communication network to reduce network energy consumption and prolong network lifetime effectively.

LEACH adopts the strategy of distributed cluster head election. Some nodes in the network become cluster heads in random ways. Although LEACH can reduce the energy consumption and prolong the network lifetime, it is lack of centralized control. LEACH can't guarantee the election of appropriate number of cluster heads, and has no medical method to ensure that the selected cluster heads are uniformly distributed in the whole network.

Ref. 6 aims at the defects of LEACH and proposes the LEACH-MAC algorithm. The algorithm is clustered according to the residual energy. LEACH-MAC selects the nodes with higher residual energy to become cluster heads one by one until the number of elected cluster heads reaches the optimum quantity. LEACH-MAC reduces energy consumption by stabilizing the number of cluster heads.

Ref. 7 tries to control the distribution of cluster heads. A node that is close to the selected cluster head cannot become cluster head. In this way, the algorithm ensures that the cluster heads are not close to each other.

LEACH and the algorithms in Ref. 6 and 7 are all single hop algorithms. If the coverage of a WSN becomes larger, the energy consumption of communication will increase substantially. Multi-hop algorithms are suitable for larger networks and DUCF is a multi-hop algorithm. DUCF elects cluster heads using fuzzy approach and limits the maximum number of nodes in a cluster to achieve load balancing [8].

ACCA is another multi-hop algorithm. ACCA select the nodes with larger residual energy, smaller distances to the around nodes and right distances to the elected cluster heads to become cluster heads [9]. 
The existing cluster selection algorithms are difficult to make a good tradeoff between energy efficiency, load balancing and other factors.

\section{PROPOSED ALGORITHM}

The model of the WSNs adopted in this paper has the following properties.

a) $N$ nodes are randomly installed in a square area with a side length of $M$.

b) The base station is fixed and has enough energy.

c) The energy of a node is limited and a node has its own residual energy information.

d) All nodes have power control capability and can change their transmission power. A node can perceive the power of the received signal and estimates the distance between the sender and receiver.

We use first order wireless communication model to calculate the energy consumption [7].

$$
\begin{aligned}
& E_{T x}(l, d)=\left\{\begin{array}{l}
l \times E_{\text {elec }}+l \times \varepsilon_{f s} d^{2}, d<d_{\text {corssover }} \\
l \times E_{\text {elec }}+l \times \varepsilon_{m p} d^{4}, d \geq d_{\text {corssover }}
\end{array}\right. \\
& E_{R x}(l)=l \times E_{\text {elec }}
\end{aligned}
$$

Where, $l$ is data sending or receiving whose unit is bit, $d$ is Communication distance. $E_{\text {elec }}$ is the energy dissipating per bit to run circuit and $E_{\text {elec }}$ is $50 \mathrm{~nJ} / \mathrm{bit}$. $\varepsilon_{f s}$ is $10 \mathrm{pJ} / \mathrm{bit} / \mathrm{m}^{2}, \varepsilon_{m p}$ is $0.0013 \mathrm{pJ} / \mathrm{bit} / \mathrm{m}^{4}$. and $d_{\text {corssover }}$ is $87 \mathrm{~m} \mathrm{[10]}$.

WSN clustering algorithm should reduce energy consumption in cluster head election and ensure the load balancing among clusters. In addition, because the cluster head is responsible for most communication, in the cluster head election the residual energy also should been taken account of. In this paper, we consider cluster head distribution, energy consumption, node residual energy and cluster load balancing when choosing cluster head.

In the multi-hop algorithm, a cluster heads near the base station transmits much information from other cluster heads, and its energy consumption is larger. In order to balance the energy consumption of cluster heads, we reduce the number of nodes in these clusters. That is to say, the clusters near the base station are smaller, and the clusters far away from the base station are larger. $R_{i}$ is the radius of cluster head $i$. We use the formula in Ref. 10 to calculate $R_{i}$. By varying the radius of the cluster heads, the nodes are divided into clusters of different sizes, so the energy balance of cluster heads is realized.

Suppose that the distance between $\mathrm{i}$ and a node in its cluster is $\mathrm{m}$. The expectation of $\mathrm{m} 2$ is $\frac{R_{i}^{2}}{2}$ [7]. The number of nodes in the circle with a radius of $\mathrm{Ri}$ and a center of $\mathrm{i}$ is ni and the average value of ni is nave. Obviously $n_{\text {ave }}=\frac{N \times \pi \times R_{i}^{2}}{M^{2}}$. This algorithm uses $\mathrm{Ci}=$ nave $\times \mathrm{E}(\mathrm{m} 2)$ as the cost parameter in communication. $\mathrm{Ci}$ too large or too small often means that there are too few nodes in the cluster or the nodes are too far from the cluster head.

When electing cluster heads, each node calculates its respective $t$ according to Eq. 2 and waits for $t$.

$$
t=\left\{\begin{array}{cc}
\left(\sum_{k=1}^{n_{i}} m_{k}^{2}-C_{i}\right)^{2} & \text { if } E_{i} \geq \mathrm{E}_{\mathrm{ave}} \\
\infty & \text { else }
\end{array}\right.
$$

Where $E_{i}$ is the residual energy of $i$ and $E_{\text {ave }}$ is the average residual energy of all living nodes.

When waiting, if $i$ receive information from a cluster head, it will recalculate $t$.

$$
t_{\text {new }}=\left\{\begin{array}{cl}
\mathrm{t}_{\text {old }}+\left(\mathrm{d}-\sqrt{2} \mathrm{R}_{\mathrm{i}}\right)^{2} & \text { if } \mathrm{d} \leq \sqrt{2} \mathrm{R}_{\mathrm{i}} \\
\mathrm{t}_{\text {old }} & \text { if } \mathrm{d}>\sqrt{2} \mathrm{R}_{\mathrm{i}}
\end{array}\right.
$$

Where $d$ is the distance between $i$ and the cluster head. The distance between two cluster heads should be moderate and cluster heads too close to each other will increase energy consumption. We increase the delay time of the nodes which are close to the elected cluster heads. By this method, the distribution of cluster heads is more reasonable.

When the delay is over, a node will become a new cluster head and broadcast a message to notify other nodes that it is a cluster head. The base station receives the messages to find how many cluster heads have been elected already. $k$ is the optimum number of cluster heads and the formula of $k$ is given in Ref.11. If $k$ cluster heads have been elected, the base station should broadcast a message to end the election. After the election, a non-cluster head node joins the cluster whose cluster head is nearest and start communication.

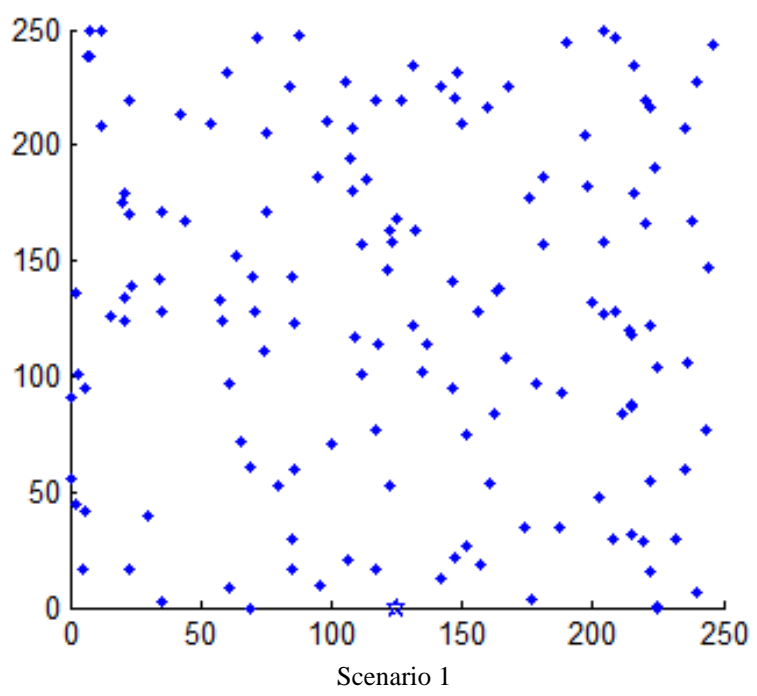




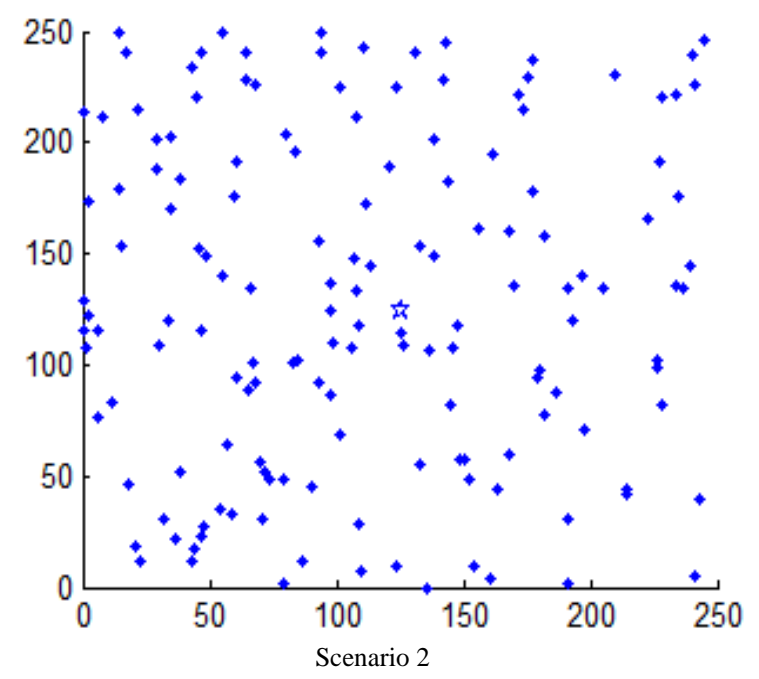

FIGURE I. NODES IN THE WSN

\section{RESULTS AND ANALYSIS}

We compared UCLB with LEACH, DUCF and ACCA. The model of the WSN is shown in Figure I and $M=250 \mathrm{~m}$, $N=150$. The points represent nodes, and the five pointed star represents the base station. The distribution of nodes and the location of the base stations are different in the two scenarios.

Energy consumption and network life cycle are important factors in evaluating network performance. The whole network energy consumptions of ten rounds are shown in Figure II. Obviously, the average distance between all nodes to the base station in scenario 2 is less than that of scenario 1 , so the energy consumptions of these algorithms in scenario 2 are lower.

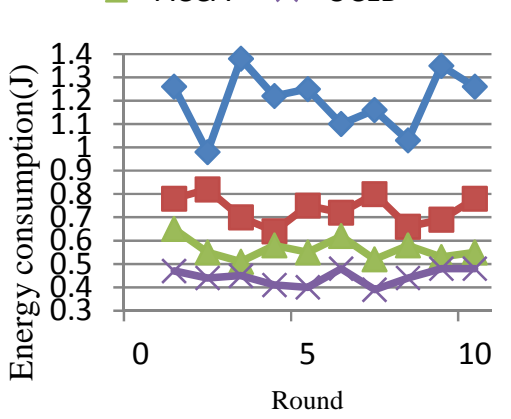

Scenario 1

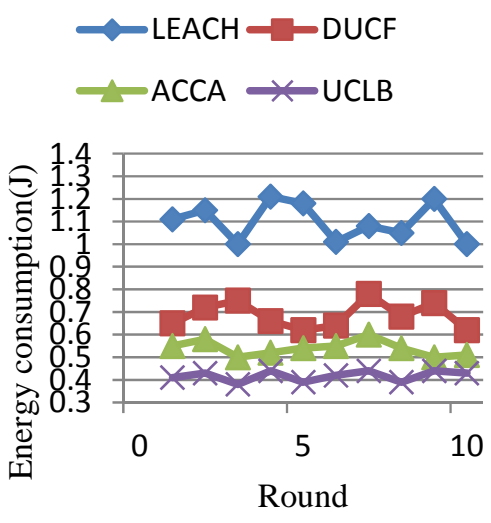

Scenario 2

FIGURE II. ENERGY CONSUMPTION

In Figure II The clustering of LEACH is completely random and it is a single hop network. When a cluster head is far away from the base station, the energy consumption of communication is large. So LEACH has the largest energy consumption and the energy consumptions of different rounds vary widely. The other three are multi-hop algorithms. If the network area is large, the energy consumption of the network is much smaller than that of LEACH.

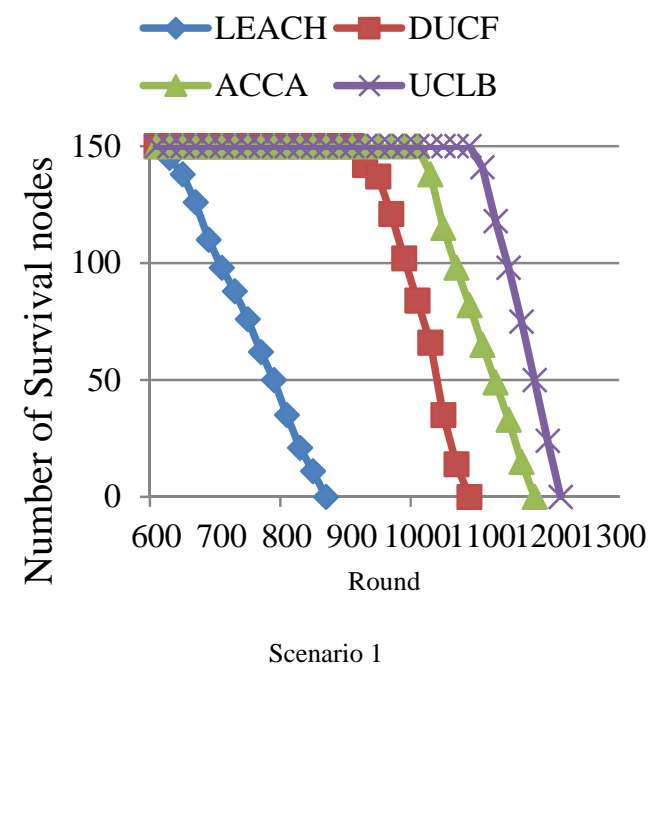




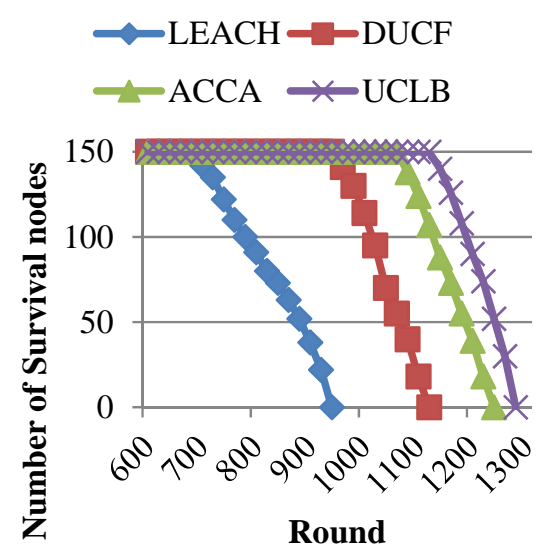

Scenario 2

FIGURE III. LIFETIME

Figure III shows the lifetimes of different algorithms. Because the energy consumption of LEACH is large, the time of all nodes death is earlier. Since LEACH does not take into account the residual energy when electing cluster heads, it is easy for some node with lower residual energy to die prematurely. The other three algorithms reduce energy consumption and ensure that nodes with lower residual energy do not become cluster heads, so the times of first node death and all nodes death are both delayed. UCLB distributes the cluster heads more reasonably and has the longest network lifetime.

\section{CONCLUSION}

UCLB is a multi-hop algorithm in which all nodes are divided into clusters of different sizes. A cost parameter is calculated by the number of surround nodes and the distance to these nodes. Delay time of each node is calculated according to the cost parameter and the distances to other cluster heads and the nodes with small delay time preferentially become cluster heads to reduce energy consumption. Further studies can be carried out on distributing the cluster heads more reasonable by other factors such as the position of all nodes.

\section{ACKNOWLEDGMENT}

The work is supported by National Natural Science Foundation of China 61527803, the National Key Scientific Instrument and Equipment Development Project 2016YFF0103702, 2016YFB1100205 and Aeronautical Science Foundation of China 2015ZF52067.

\section{REFERENCES}

[1] Prabhu, B., Gajendran, E., \& Balakumar, N. (2017). Prospective applications of wireless sensor node and wireless sensor network. Social Science Electronic Publishing.

[2] Fummi, F., Perbellini, G., Acquaviva, A., Quaglia, D., \& Sensornetzwerke, D. (2017). Wireless Sensor Network Architectures. Springer, Berlin.

[3] Ennajari, H., Maissa, Y. B., \& Mouline, S. (2017). Energy Efficient InNetwork Aggregation Algorithms in Wireless Sensor Networks: A Survey. Advances in Ubiquitous Networking 2. Springer Singapore, $135-148$.
[4] Singh, K. (2015). WSN LEACH based protocols: A structural analysis. International Conference and Workshop on Computing and Communication.

[5] Heinzelman, W. R., Chandrakasan, A., \& Balakrishnan, H. (2000). Energy-Efficient Communication Protocol for Wireless Microsensor Networks. Hawaii International Conference on System Sciences (Vol.18, pp.8020). IEEE Computer Society.

[6] Batra, P. K., \& Kant, K. (2016). Leach-mac: a new cluster head selection algorithm for wireless sensor networks. Wireless Networks, 22(1), 1-12.

[7] Jia Y, Zhang C, Liang K. (2017). A Distributed Multi-competitive Clustering Approach for Wireless Sensor Networks. International Journal of Wireless Information Networks:1-8.

[8] Baranidharan, B., \& Santhi, B. (2016). Ducf: distributed load balancing unequal clustering in wireless sensor networks using fuzzy approach. Applied Soft Computing, 40, 495-506.

[9] Afsar, M., Tayarani-N, M. H., \& Aziz, M. (2016). An adaptive competition-based clustering approach for wireless sensor networks. Telecommunication Systems, 61(1), 181-204.

[10] Xia, H., Zhang, R. H., Yu, J., \& Pan, Z. K. (2016). Energy-efficient routing algorithm based on unequal clustering and connected graph in wireless sensor networks. International Journal of Wireless Information Networks, 23(2), 1-10.

[11] Sun, Y. Q., Peng, J., Liu, T., \& Chen, X. H. (2014). Uneven clustering routing protocol based on dynamic partition for wireless sensor network. Journal on Communications, 35, 198-206. 